\title{
Analysis of the guidance value of 3D ultrasound in evaluating endometrial receptivity for frozen-thawed embryo transfer in patients with repeated implantation failure
}

\author{
Ruiqing Tong, Ying Zhou, Qi He, Yanyan Zhuang, Weiqin Zhou, Fei Xia \\ Reproductive Medicine Center of the First Affiliated Hospital of Soochow University, Suzhou 215006, China \\ Contributions: (I) Conception and design: R Tong; (II) Administrative support: F Xia; (III) Provision of study materials or patients: R Tong, Y Zhou; (IV) \\ Collection and assembly of data: Q He, W Zhou; (V) Data analysis and interpretation: R Tong, Y Zhuang; (VI) Manuscript writing: All authors; (VII) \\ Final approval of manuscript: All authors. \\ Correspondence to: Fei Xia. Reproductive Medicine Center of the First Affiliated Hospital of Soochow University, Suzhou 215006 , China. \\ Email: feix0513@126.com.
}

Background: Repeated implantation failure (RIF) is currently believed by some scholars to be mainly related to the poor endometrial receptivity. Three-dimensional (3D) ultrasound, as a noninvasive examination, has attracted the most attention. This paper further discusses whether 3D ultrasound of the endometrial receptivity index has guidance value in the evaluation of pregnancy outcomes in patients with RIF following frozen-thawed embryo transfer.

Methods: A total of 79 patients with RIF were retrospectively analyzed. These 79 patients which were confirmed by the transvaginal ultrasonography detection comprised 36 cases of intrauterine pregnancy, which were included in the pregnancy group, and 43 cases with biochemical pregnancy (the HCG in blood or urine is only transient and can be detected by clinical biochemical methods. The pregnancy sac cannot be seen under ultrasound) or negative results of human chorionic gonadotropin (HCG) examination, which were included in the non-pregnancy group. The endometrial thickness, uterine volume, sub-endometrial blood flow type, pulsatility index (PI) and resistance index (RI) of the spiral artery; the RI and PI of the uterine artery; and the peak systolic velocity/end diastolic velocity (S/D) of the two groups were measured and analyzed.

Results: (I) There were no significant differences in the age, infertility years, body mass index (BMI), anti-Müllerian hormone (AMH), endometrial thickness on the hCG injection day, estradiol (E2), and progesterone $(\mathrm{P})$ between the pregnant and non-pregnant groups $(\mathrm{P}>0.05)$. (II) There were no significant differences in the endometrial thickness, uterine volume, and RI and PI of the uterine artery on the day before the implantation between the two groups $(\mathrm{P}>0.05)$. (III) There was statistical difference in the sum of the $\mathrm{S} / \mathrm{D}$ of the two uterine arteries between the two groups $(\mathrm{P}<0.05)$ with a cutoff value of $14.47(\mathrm{P}<0.05)$. (IV) The RI and PI of the spiral artery in the non-pregnancy group were lower than those in the pregnancy group, and the difference was statistically significant $(\mathrm{P}<0.05)$. (V) The differences in the endometrial classification and subendometrial blood flow classification between the two groups were statistically significant $(\mathrm{P}<0.05)$.

Conclusions: Ultrasonographic endometrial blood flow classification, spiral artery blood flow parameters, and uterine artery blood flow parameters can be effective indexes for evaluating endometrial receptivity, and they have a certain clinical significance in evaluating the pregnancy outcome of RIF patients after retransplantation.

Keywords: 3D ultrasound; endometrial receptivity; repeated implantation failure (RIF); frozen-thawed embryo transfer 
Submitted Jun 21, 2020. Accepted for publication Aug 04, 2020.

doi: $10.21037 /$ atm-20-5463

View this article at: http://dx.doi.org/10.21037/atm-20-5463

\section{Introduction}

Repeated implantation failure (RIF) refers to a failure to achieve clinical pregnancy after transfer of at least four good-quality embryos in a minimum of three fresh or frozen cycles in a woman under the age of 40 years $(1,2)$. The causes for RIF are very complex and generally involve factors related to the embryo and endometrium. RIF is currently believed by some scholars to be mainly related to poor endometrial receptivity. Clinically, the common factors that affect endometrial receptivity are hydrosalpinx, endometriosis, adenomyosis, endometritis, hysteromyoma, and the change of endogenous hormone level caused by superovulation. Current methods of abortion treatment include anticoagulant therapy, immunosuppression, immunoregulation and gene screening, etc. At present, the main methods to evaluate endometrial receptivity include endometrial receptivity-related markers (such as pinocytosis), endometrial receptivity factors, and ultrasonography. Among these, three-dimensional (3D) ultrasound, as a non-invasive examination, has received more attention in evaluating endometrial receptivity by virtue of its ability to monitor endometrial thickness, endometrial peristalsis wave, endometrial typing, volume ultrasound, and endometrial and subendometrial blood flow parameters. It is generally believed that endometrial volume measurement is more accurate than endometrial thickness in evaluating endometrial receptivity and that it is a good index to predict endometrial receptivity. In this study, there was no significant difference. In order to further explore the value of $3 \mathrm{D}$ ultrasound in evaluating endometrial receptivity in patients with RIF following frozen-thawed embryo transfer, this study retrospectively analyzed the data of 79 patients with RIF who received frozen-thawed embryo transfer in our center from January 2018 to December 2019. We further explored whether 3D ultrasound of the endometrial receptivity index had guidance value in the evaluation of pregnancy outcome of the patients with RIF following frozen-thawed embryo transfer.

We present the following article in accordance with the MDAR reporting checklist (available at http://dx.doi. org/10.21037/atm-20-5463).

\section{Methods}

All procedures performed in this study involving human participants were in accordance with the Declaration of Helsinki (as revised in 2013). This study was approved by Medical ethics committee of the First Affiliated Hospital of Suzhou University (No. 137). Because of the retrospective nature of the research, the requirement for informed consent was waived.

\section{Research subjects}

A retrospective analysis was made of 79 patients with RIF who received frozen-thawed embryo transfer in the Reproductive Medicine Center of the First Affiliated Hospital of Soochow University from January 2018 to December 2019 and met the inclusion and exclusion criteria of this study. Among them, 36 cases of intrauterine pregnancy indicated by transvaginal ultrasound $30-$ 35 days after transplantation were included in the pregnancy group and 43 cases with negative serum human chorionic gonadotropin (hCG) or short-term increase of serum hCG (biochemical pregnancy) 2 weeks after the transplantation were included in the non-pregnancy group.

\section{Inclusion criteria}

(I) Age $<38$ years, (II) regular menstrual cycle, (III) natural cycle or letrozole ovulation induction cycle used in endometrial preparation scheme, (IV) at least one highquality embryo of the two embryos transplanted on the third day, and $(\mathrm{V})$ meeting the definition of RIF.

\section{Exclusion criteria}

(I) Autoimmune diseases or prethrombotic state; (II) uterine or uterine cavity occupation disease such as uterine myoma, adenomyomas, uterine polyps, submucous myomas, or adhesion of uterine cavity, etc.; (III) uniangular uterine malformation, double uterine malformation, etc.; (IV) hydrosalpinx according to B-ultrasound or hysterosalpingography (HSG); (V) pelvic endometriosis; 
and (VI) chromosomal abnormality of either spouse.

\section{Research methods}

Endometrial preparation, luteal support, and pregnancy outcome determination

Endometrial preparation and transplantation time: natural cycle or letrozole ovulation induction cycle was used for all cases. Ovulation monitoring started from the $10^{\text {th }}$ day of the cycle in the patients with a regular menstrual cycle, and ovulation induction was performed by orally taking letrozole from the $3^{\text {rd }}$ day of the cycle in the patients with irregular menstrual cycle or anovulation. When the diameter of the dominant follicle was more than 16-18 mm and the thickness of the endometrium was at least $6 \mathrm{~mm}$, the levels of serum luteinizing hormone (LH), estradiol (E2), and progesterone $(\mathrm{P})$ were measured. To induce ovulation, when $\mathrm{E} 2$ was more than $100 \mathrm{pg} / \mathrm{mL}$ and $\mathrm{P}$ was less than $1.5 \mathrm{ng} / \mathrm{mL}$, hCG 10,000 units were injected (IM) in the afternoon or at 9:00 p.m. on the same day according to the level of LH. Two days later, Duphaston $20 \mathrm{mg}$ was taken orally twice a day for 3 days to transform the endometrium, and frozen-thawed embryos transfer was performed under the guidance of ultrasound 5 days later. Two D3 embryos were transferred, with at least one being of high quality.

\section{Luteal support scheme}

The patients were given Duphaston $20 \mathrm{mg}$ twice a day orally and a $200 \mathrm{mg}$ (micronized) progesterone soft capsule twice a day through intravaginal tamponade. The administration started from the day the transplantation was made and lasted for 14 days.

\section{Pregnancy outcome determination}

The serum hCG level was measured 14 days after transplantation. The patients with negative serum hCG or whose serum hCG was temporarily increased and turned negative (biochemical pregnancy) were all placed into the non-pregnancy group, while those patients with a pregnancy sac and yolk sac under B-ultrasound 30-35 days after transplantation were placed into the pregnancy group.

\section{D ultrasonic examination method}

3D ultrasound was performed by the same senior ultrasound doctor on the day before transplantation for all patients who were prepared for frozen-thawed embryo transfer. After emptying their bladder, the patients assumed the cystolithotomy position and breathed calmly. A GE Voluson
E8 color Doppler ultrasound diagnostic instrument (Medical Electronics Company Inc.) and a RIC-9-D intracavity probe at frequency of $10 \mathrm{MHz}$ were used. 3D ultrasound monitoring parameters included endometrial thickness and classification endometrial and subendometrial blood flow classification parameters, spiral artery blood flow parameters [pulsatility index $(\mathrm{PI})$, and resistance index $(\mathrm{RI})]$, uterine artery blood flow parameters (PI and RI, and their mean values of the two sides, $\mathrm{mRI}$ and $\mathrm{mPI}$ respectively), the peak systolic velocity/ end diastolic velocity (S/D), and the volume of the uterine cavity in three dimensions. In this study, the endometrium was classified according to the 3-grade system proposed by Gonen et al. (3): type A, trilinear endometrium; type B and type C, no trilinear endometrium. Subendometrial blood flow was classified into three types based on the distribution of blood flow in endometrium and subendometrium according to the method introduced by Applebaum (4): type I, the vessels pass through the lateral hypo-echoic band of the endometrium, but do not enter the hyper-echoic rim of the endometrium; type II, the vessels pass through the hyper-echoic rim of the endometrium, but do not enter the endometrium; type III, the vessels enter the endometrium.

\section{Statistical analysis}

The clinical data and ultrasonic parameters were analyzed by SPSS 23.0 statistical software. The measurement data was compared with $t$ test, and the results are expressed by mean \pm standard deviation. When the $\mathrm{P}$ value $<0.05$, the difference was considered statistically significant. $\chi^{2}$ test was used for the counting data, and the results are expressed in percentages. The area under receiver operating characteristic (ROC) curve was calculated for the statistically significant parameters to predict pregnancy outcome.

\section{Results}

\section{Analysis of the general data of the research subjects:}

There was no significant difference in the age, infertility years, body mass index (BMI) and other basic conditions except between the non-pregnant group and the pregnant group $(\mathrm{P}>0.05)$ (see Table 1).

\section{Hormone level, endometrium thickness, and classification of the two groups on the day of HCG injection}

There was no significant difference in E2 and P levels 
Table 1 Comparison of general conditions between the non-pregnant group and pregnant group

\begin{tabular}{lccc}
\hline Characteristics & Non-pregnancy group $(\mathrm{n}=43)$ & Pregnancy group $(\mathrm{n}=36)$ & $\mathrm{P}$ \\
\hline Age (years) & $32.53 \pm 4.62$ & $31.0 \pm 4.18$ & 0.13 \\
Year of infertility & $4.13 \pm 2.73$ & $3.72 \pm 2.80$ & 0.51 \\
$\mathrm{BMI}\left(\mathrm{kg} / \mathrm{m}^{2}\right)$ & $22.30 \pm 2.91$ & $21.5 \pm 2.32$ & 0.21 \\
$\mathrm{AMH}(\mathrm{ng} / \mathrm{mL})$ & $5.28 \pm 4.36$ & $5.06 \pm 3.35$ & 0.79 \\
hCG thickness $(\mathrm{mm})$ & $9.69 \pm 1.97$ & $10.30 \pm 2.39$ & 0.23 \\
hCG $\mathrm{E}_{2}(\mathrm{pg} / \mathrm{mL})$ & $330.32 \pm 110.79$ & $326.6 \pm 164.02$ & 0.90 \\
hCG $\mathrm{P}(\mathrm{ng} / \mathrm{mL})$ & $0.60 \pm 0.45$ & $0.54 \pm 0.45$ & 0.55 \\
\hline
\end{tabular}

hCG thickness represents endometrium thickness on the day hCG injection; hCG $E_{2}$ represents $E_{2}$ on the day of hCG injection; hCG $P$ represents $P$ on the day of hCG injection; hCG endometrium pattern represents endometrium pattern on the day of hCG injection; the difference was statistically significant if $\mathrm{P}<0.05$. BMI, body mass index; $\mathrm{AMH}$, anti-Müllerian hormone; hCG, human chorionic gonadotropin; $E_{2}$, estradiol; $P$, progesterone.

between the pregnant group and the non-pregnant group on the day of HCG injection ( $\mathrm{P}>0.05)$. On the day of HCG injection, the endometrial thickness in the pregnant group $(10.30 \pm 2.39 \mathrm{~mm})$ was slightly thicker than that in the nonpregnant group $(9.69 \pm 1.97 \mathrm{~mm})$, but the difference was not statistically significant $(\mathrm{P}>0.05)$ (see Table 1).

\section{Comparison of endometrial receptive ultrasound parameters between two the groups one day before transplantation}

There was no significant difference in endometrial thickness and uterine cavity volume (see Figure 1) between the pregnant group and the non-pregnant group $(\mathrm{P}>0.05)$. There was no significant difference in the mean blood flow parameters (see Figure 2) of the uterine artery between the two groups $(\mathrm{P}>0.05)$ (see Table 2$)$.

The RI $(0.51 \pm 0.14)$ and PI $(0.45 \pm 0.11)$ (see Figure 3$)$ of the spiral artery in the non-pregnant group were lower than those in the pregnant group (RI, $0.72 \pm 0.18$; PI, $0.65 \pm 0.11$ ), and the difference between the two groups was statistically significant $(\mathrm{P}<0.05)$. The sum of $\mathrm{S} / \mathrm{D}$ of the two uterine arteries in the pregnancy group $(12.29 \pm 2.46)$ was lower than that in the non-pregnancy group $(16.33 \pm 12.56)$, and the difference between the two groups was statistically significant $(\mathrm{P}<0.05)$. The cutoff of the sum of $\mathrm{S} / \mathrm{D}$ of the two uterine arteries was 14.47 and showed statistical significance $(\mathrm{P}<0.05)$. The area under ROC curve of the S/D was 0.59. See Table 2 and Figures 4 for details.

\section{Discussion}

With the development and application of assisted reproductive technology in China over the last 30 years, the embryo implantation rate and clinical pregnancy rate of assisted reproductive technology has been significantly improved. However, $5-10 \%$ of the women who receive in vitro fertilization (IVF) or intracytoplasmic sperm injection (ICSI) treatment have RIF (5). Indeed, a significant number of frozen-thawed embryo transfer patients who have no anatomical abnormalities in the reproductive system, hydrosalpinx, adenomyosis, prethrombotic state, or abnormal immune factors, still experience of RIF. At present, this is generally considered to be related to poor endometrial receptivity. As endometrial receptivity (6) refers to the ability of the endometrium to allow normal embryo implantation, optimal endometrial receptivity provides a normal implantation opportunity for a stable pregnancy, through good adhesion, invasion, and implantation. This specific time is also called the "implantation window" (7). How to evaluate endometrial receptivity and avoid implantation failure from inaccurate endometrial evaluation is a crucial problem (8). The current methods for evaluating endometrial receptivity include related markers of endometrial receptivity (e.g., Pinopodes), endometrial receptive factors (e.g., interleukin, epidermal growth factor, vascular endothelial growth factor, estrogen receptor, progesterone receptor), and ultrasonography. The detection of pinopodes and endometrial receptive factors has limited application in clinical practice due to its high cost and invasive nature. As a timely, economical, and noninvasive examination, transvaginal $3 \mathrm{D}$ ultrasound has received increased attention due to its advantages in evaluating endometrial receptivity. Generally, endometrial receptivity is evaluated based on the endometrial thickness, 


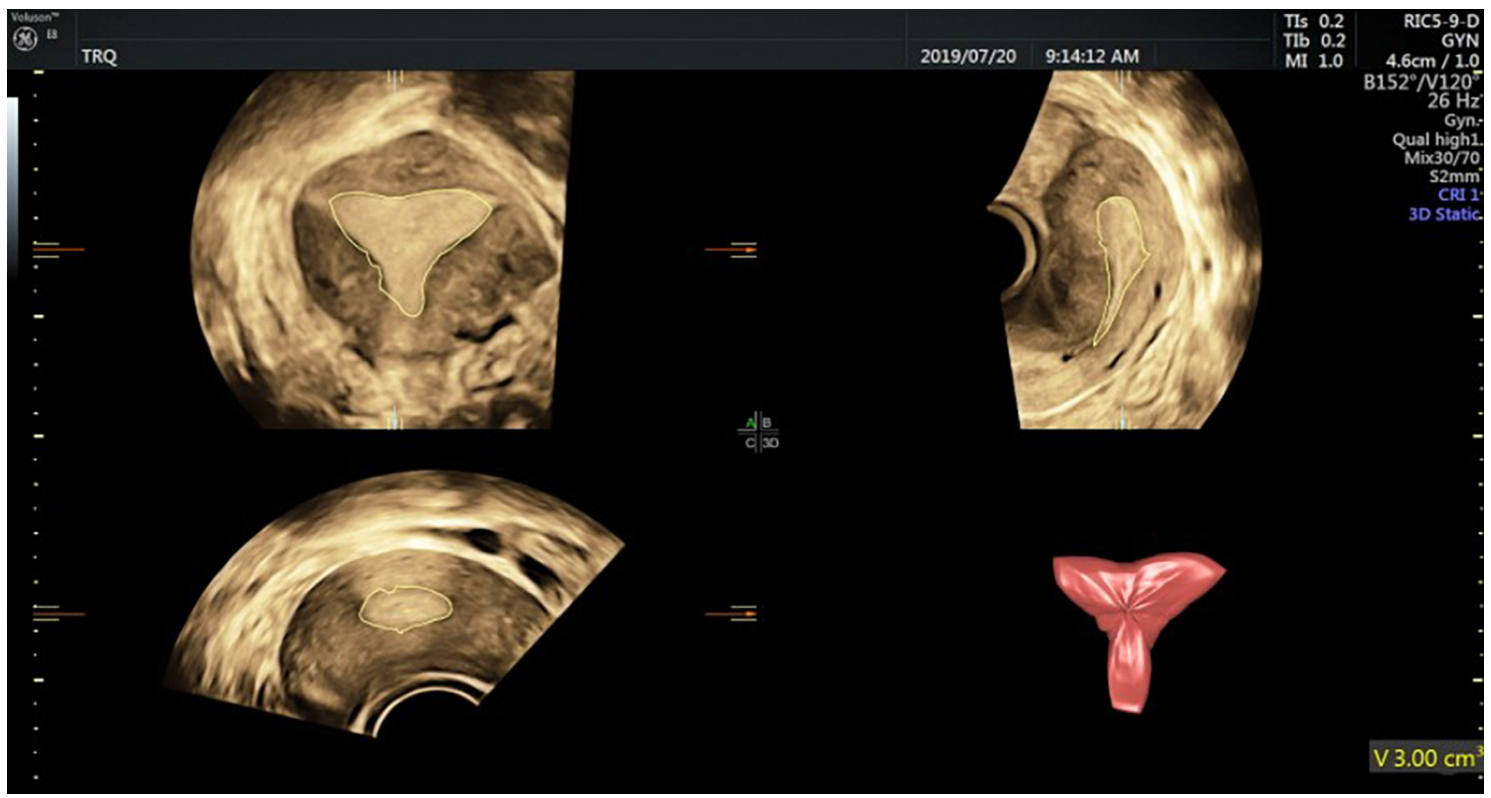

Figure 1 Uterine cavity volume.

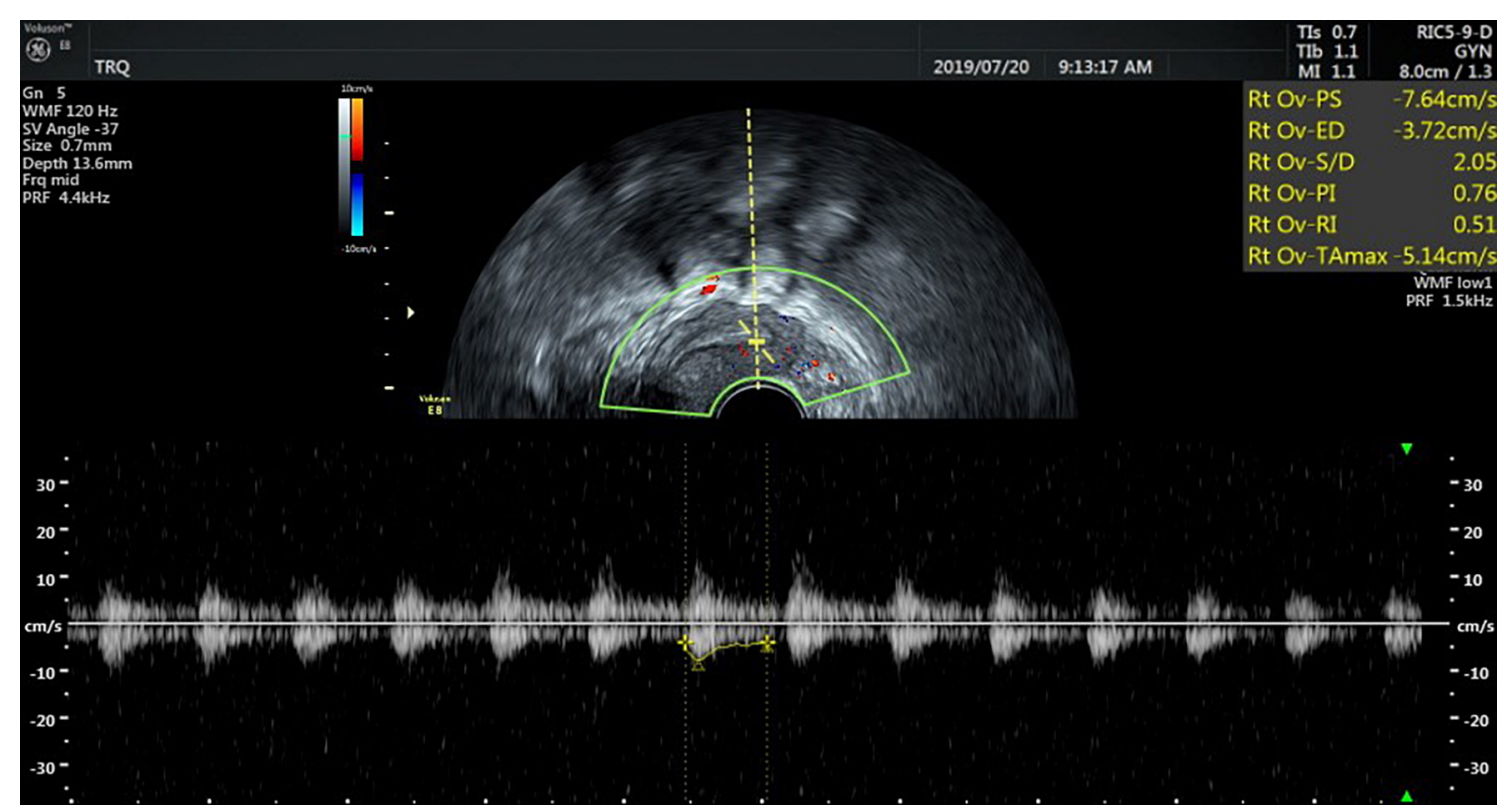

Figure 2 Blood flow spectrum of the uterine spiral artery.

endometrial classification, uterine cavity volume, and endometrial and subendometrial blood supply $(9,10)$.

Whether endometrial thickness can predict the outcome of pregnancy remains controversial. Some studies have shown that trilinear pattern with moderate endometrial thickness may be related to a good clinical outcome. When endometrial thickness is less than $7 \mathrm{~mm}$ or more than $14 \mathrm{~mm}$, the combination of endometrial thickness and endometrial morphology cannot predict the outcome of in vitro fertilization and embryo transfer (IVF-ET) (11). Currently, it is generally recognized that endometrial thickness $<8 \mathrm{~mm}$ has a strong negative predictive value (12). 
Table 2 Comparison of endometrial thickness, uterine cavity volume, and uterine spiral artery and uterine artery blood flow parameters between the non-pregnancy group and pregnancy group

\begin{tabular}{lcc}
\hline Parameters & Non-pregnancy group $(\mathrm{n}=43)$ & Pregnancy group $(\mathrm{n}=36)$ \\
\hline En thickness $(\mathrm{mm})$ & $9.50 \pm 2.21$ & $10.21 \pm 2.52$ \\
Ut volume $(\mathrm{mL})$ & $4.08 \pm 2.02$ & $4.68 \pm 2.48$ \\
Spiral artery & & \\
RI & $0.51 \pm 0.14$ & $0.72 \pm 0.18$ \\
PI & $0.45 \pm 0.11$ & $0.65 \pm 0.11$ \\
Uterine artery & & \\
mRI & $0.84 \pm 0.55$ & $0.80 \pm 0.44$ \\
mPI & $2.31 \pm 0.48$ & $2.22 \pm 0.36$ \\
Sum of S/D & $16.33 \pm 12.56$ & $12.29 \pm 2.46$ \\
Sub blood flow pattern & & $0.03^{*}$ \\
Type I & $17(39.53 \%)$ & 0.37 \\
Type II & $14(32.56 \%)$ & $9(25 \%)$ \\
Type III & $12(27.91 \%)$ & $6(16.67 \%)$ \\
\hline
\end{tabular}

En thickness, endometrium thickness; Ut volume, uterine cavity volume; sub blood flow pattern, subendometrial blood flow pattern; PI, pulsatility index; RI, resistance index; S/D, the peak systolic velocity/end diastolic velocity.

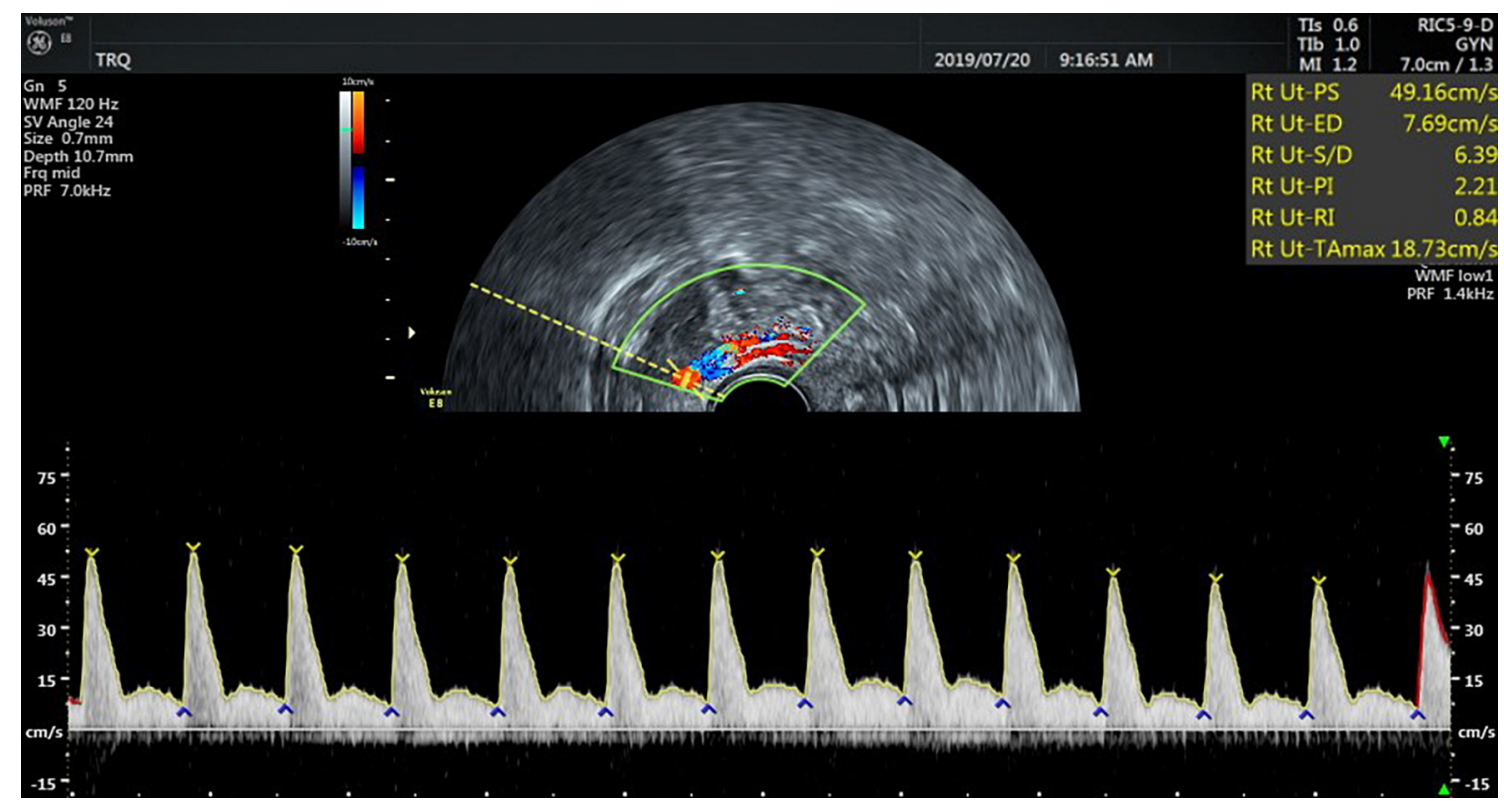

Figure 3 Blood flow spectrum of the uterine artery.

Given the potential dynamic relationship between endometrial thickness and estrogen level in the menstrual cycle, this study chose to measure the endometrial thicknesses in the day of hCG injection and on the day before frozen-thawed embryo transfer. Results suggested that the endometrial thickness of the pregnancy group was slightly greater than that of the non-pregnancy group, but there was no statistical difference $(P>0.05)$ between the 


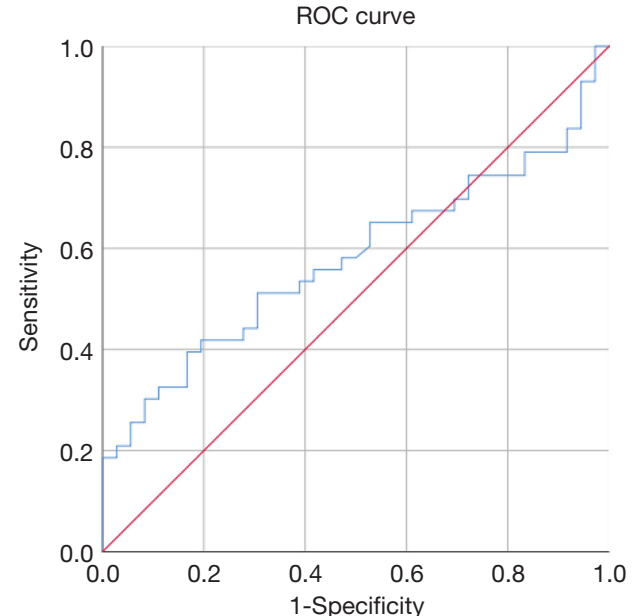

Figure 4 The area under the ROC curve of S/D of the uterine artery. ROC, receiver operating characteristic; S/D, the peak systolic velocity/end diastolic velocity.

two groups. There were 8 cases $(18.6 \%)$ with endometrial thickness less than $8 \mathrm{~mm}$ in the non-pregnancy group, and 6 cases $(16.17 \%)$ with endometrial thickness less than $8 \mathrm{~mm}$ in the pregnancy group. There was no significant difference in the endometrial thickness between the two groups. This result suggests that implantation should be avoided in RIF patients with thinner endometria. It was also found that the ratio of trilinear endometrium in the non-pregnancy group was significantly lower than that in the pregnancy group $(\mathrm{P}<0.05)$. Taken together, the findings indicate that endometrial typing can predict the pregnancy outcome of RIF patients and can be used as one of the main indicators of endometrial receptivity in RIF patients.

It is generally believed that endometrial volume measurement is more accurate than endometrial thickness in evaluating endometrial receptivity and that it is a good index to predict endometrial receptivity. Most studies support the notion that endometrial volume must reach $2.0-2.5 \mathrm{~mL}$ to support a pregnancy; the pregnancy rate is significantly reduced if the volume is less than $3.0 \mathrm{~mL}$, and the pregnancy rate is 0 if the volume is less than $1.0 \mathrm{~mL}$ (13). In this study, the uterine volume of the pregnant group was slightly higher than that of the non-pregnant group, but there was no significant difference between the two groups. There were two cases whose volume was between $1.0-1.5 \mathrm{~mL}$, but the spiral artery showed small resistance, and clinical pregnancy was achieved.

Endometrial and subendometrial blood perfusion is necessary for embryo implantation. The parameters of endometrial and subendometrial blood flow mainly include the RI, PI, and S/D of uterine artery; the RI and PI of the spiral artery; and subendometrial blood flow classification. When RIs and PIs are low, it means endometrial blood perfusion is good, with the opposite indicating poor blood perfusion. Some studies have shown that the uterine blood flow has the highest resistance index in the proliferative period, begins to decrease 1 day before ovulation, reaches the lowest point on the $18^{\text {th }}$ day, and remains at this level for the rest of the cycle. These changes do not occur during the anovulatory cycle (14). Therefore, we chose to measure the parameters of endometrial blood flow on the day before transplantation (equivalent to about 18 days of menstruation). Some studies have shown that there is no correlation between clinical pregnancy outcome and the PI and S/D of the uterine artery, but there is a negative correlation with RI and a positive correlation with the distribution of subintimal microvasculature (15). In this study, we also found that there was no significant difference in the PI, RI, and S/D of the uterine arteries between the two groups $(\mathrm{P}>0.05)$, but the sum $\mathrm{S} / \mathrm{D}$ in the pregnant group was significantly lower than that in the non-pregnant group $(\mathrm{P}<0.05)$ with a cutoff of 14.47 . When $\mathrm{S} / \mathrm{D}$ was more than 14.47 , it had the statistically significant ability to predict the clinical pregnancy rate $(\mathrm{P}<0.05)$; however, the predictive significance should be interpreted cautiously as the area under the ROC curve was only 0.59 .

The blood vessels in the myometrium gradually become branches of the spiral artery with endometrial hyperplasia, which is the main source of endometrial blood supply. Therefore, the blood flow parameters of spiral artery may be a better index for evaluating endometrial receptivity. In this study, the RI and PI of the spiral artery in the pregnancy group were lower than those in the nonpregnancy group, the ratio of type III endometria in the pregnancy group was significantly higher than that in the non-pregnancy group, and these differences between the two groups were statistically significant. We also found that the S/D and PI of recurrent spontaneous abortion (RSA) patients were significantly higher than those of normal pregnant women. It is believed that the increased resistance of the blood flow of the uterine artery leads to the decrease of the degree of endometrial vascularization, thus affecting the blood circulation between the mother and fetus along with the uterine blood flow (16). It is also believed that the increased resistance of uterine artery and spiral artery in RIF patients can reduce the blood perfusion of the endometrium and sub endometrium, which is not conducive 
to embryo implantation and thus may contribute to RIF. Therefore, the blood flow resistance of RIF patients should be reduced as much as possible to improve the pregnancy rate.

In conclusion, properly evaluating endometrial receptivity and selecting the right time for transplantation are critical to improving the clinical pregnancy rate of RIF patients. For RIF patients, 3D ultrasound, as a noninvasive and rapid means for monitoring endometrial and subendometrial blood flow parameters, may be a simple and effective method for evaluating uterine receptivity.

\section{Acknowledgments}

Funding: This study was supported by Provincial Key Clinical Specialty (Ultrasound Medicine Department) of Jiangsu Province, China (2019001308060009).

\section{Footnote}

Reporting Checklist: The authors have completed the MDAR reporting checklist. Available at http://dx.doi.org/10.21037/ atm-20-5463

Data Sharing Statement: Available at http://dx.doi. org/10.21037/atm-20-5463

Conflicts of Interest: All authors have completed the ICMJE uniform disclosure form (available at http://dx.doi. org/10.21037/atm-20-5463). The authors have no conflicts of interest to declare.

Ethical Statement: The authors are accountable for all aspects of the work in ensuring that questions related to the accuracy or integrity of any part of the work are appropriately investigated and resolved. All procedures performed in this study involving human participants were in accordance with the Declaration of Helsinki (as revised in 2013). This study was approved by Medical ethics committee of the First Affiliated Hospital of Suzhou University (No. 137). Because of the retrospective nature of the research, the requirement for informed consent was waived.

Open Access Statement: This is an Open Access article distributed in accordance with the Creative Commons Attribution-NonCommercial-NoDerivs 4.0 International License (CC BY-NC-ND 4.0), which permits the noncommercial replication and distribution of the article with the strict proviso that no changes or edits are made and the original work is properly cited (including links to both the formal publication through the relevant DOI and the license). See: https://creativecommons.org/licenses/by-nc-nd/4.0/.

\section{References}

1. Coughlan C, Ledger W, Wang Q, et al. Recurrent implantation failure: definition and management. Reprod Biomed Online 2014;28:14-38.

2. Sharma N. GnRH agonist and letrozole in women with recurrent implantation failure. Ann Transl Med 2019;7:S209.

3. Gonen Y, Casper RF, Jacobson W, et al. Endometrial thickness and growth during ovarian stimulation: a possible predictor of implantation in in vitro fertilization. Fertil Steril 1989;52:446-50.

4. Applebaum M. The uterine biophysical profile. Ultrasound Obstet Gynecol 1995;5:67-8.

5. Mak JSM, Chung CHS, Chung JPW, et al. The effect of endometrial scratch on natural-cycle cryopreserved embryo transfer outcomes: a randomized controlled study. Reprod Biomed Online 2017;35:28-36.

6. Lessey BA, Young SL. What exactly is endometrial receptivity? Fertil Steril 2019;111:611-7.

7. Karizbodagh MP, Rashidi B, Sahebkar A, et al. Implantation Window and Angiogenesis. J Cell Biochem 2017;118:4141-51.

8. Cha J, Sun X, Dey SK. Mechanisms of implantation: strategies for successful pregnancy. Nat Med 2012;18:1754-67.

9. Cheng F, Li T, Wang QL, et al. Effects of hydrosalpinx on ultrasonographic parameters for endometrial receptivity during the window of implantation measured by power color Doppler ultrasound. Int J Clin Exp Med 2015;8:6103-8.

10. Chen M, He Y, Zhang P, et al. Comparison of Uterine Receptivity between Fertile and Unexplained Infertile Women by Assessment of Endometrial and Subendometrial Perfusion Using Contrast-Enhanced Ultrasound: Which Index is Better--Peak Intensity or Area under the Curve? Ultrasound Med Biol 2016;42:654-63.

11. Zhao J, Zhang Q, Li Y. The effect of endometrial thickness and pattern measured by ultrasonography on pregnancy outcomes during IVF-ET cycles. Reprod Biol Endocrinol 2012;10:100.

12. Richter KS, Bugge KR, Bromer JG, et al. Relationship between endometrial thickness and embryo implantation, 
based on 1,294 cycles of in vitro fertilization with transfer of two blastocyst-stage embryos. Fertil Steril 2007;87:53-9.

13. Zollner U, Zollner KP, Specketer MT, et al. Endometrial volume as assessed by three-dimensional ultrasound is a predictor of pregnancy outcome after in vitro fertilization and embryo transfer. Fertil Steril 2003;80:1515-7.

14. Kurjak A, Kupesic-Urek S, Schulman H, et al. Transvaginal color flow Doppler in the assessment of ovarian and uterine blood flow in infertile women. Fertil Steril 1991;56:870-3.

Cite this article as: Tong R, Zhou Y, He Q, Zhuang Y, Zhou W, Xia F. Analysis of the guidance value of 3D ultrasound in evaluating endometrial receptivity for frozen-thawed embryo transfer in patients with repeated implantation failure. Ann Transl Med 2020;8(15):944. doi: 10.21037/atm-20-5463
15. Li Z, Wang X, Guan Y, et al. Uterine artery blood flow and microvessel density by vaginal color Doppler ultrasonography in embryo implantation failure. Exp Ther Med 2017;14:4797-800.

16. Jamal A, Abbasalizadeh F, Vafaei H, et al. Multicenter screening for adverse pregnancy outcomes by uterine artery Doppler in the second and third trimester of pregnancy. Med Ultrason 2013;15:95-100.

(English Language Editor: J. Gray) 